\title{
El observatorio regional de la primera infancia
}

\author{
María Nuria Rodríguez de Martínez \\ Universidad Autónoma de Bucaramanga, Colombia \\ mrodriguez4@unab.edu.co \\ Socorro Astrid Portilla Castellanos \\ Universidad Autónoma de Bucaramanga, Colombia \\ sportilla2@unab.edu.co \\ Alhim Adonaí Vera Silva \\ Universidad Autónoma de Bucaramanga, Colombia \\ avera6@unab.edu.co
}

\begin{abstract}
Resumen
El Observatorio Regional de la Primera Infancia es fruto del estudio financiado por la Universidad Autónoma de Bucaramanga para examinar la educación de los niños menores de seis años en la ciudad de Bucaramanga y su Área Metropolitana. La investigación permitió analizar situaciones, fenómenos, circunstancias y tendencias de la realidad educativa de la niñez atendida en instituciones o centros educativos, públicos, privados, fundaciones y ONG. En este análisis se identificaron cuatro áreas prioritarias relacionadas con la educación de la primera infancia: 1). Calidad en el desarrollo de los procesos educativos, 2). Formación de los docentes que atienden los niños, 3). Vinculación de las familias a los procesos formativos y 4). Calidad de los programas educativos que orientan el quehacer pedagógico de las instituciones.
\end{abstract}

Palabras Clave: Observatorio Regional, Primera infancia, calidad de la educación y procesos pedagógicos.

\section{The Regional Centre for Early Childhood}

\begin{abstract}
The Regional Centre for Early Childhood is the result of the study funded by the Universidad Autónoma de Bucaramanga to examine the education of children under six years in the city of Bucaramanga and her Metropolitan Area. The research allowed us to analyze situations, phenomena, circumstances and trends of the educational reality of children that are cared in educations institutions or centers, public and private institutions, foundations and ONG. In this analysis four priority areas related to early childhood education were identified. 1) Quality in the development of educational processes, 2). Training teachers serving children, 3). Linking families to training processes and 4) Quality of educational programs that guide the pedagogical work of the institutions.
\end{abstract}

Keywords: The Regional Centre, Early Childhood, Quality of education and Pedagogic processes. 


\section{Calidad en el desarrollo de los procesos}

El niño menor de seis años tiene en este lapso de tiempo la mayor oportunidad para su desarrollo cognitivo, afectivo, psicomotor y comunicativo. En consecuencia, la educación Preescolar ha de garantizar que éste desarrollo se dé en todas las instituciones que trabajan con niños de cero a cinco años. Hace ya varios años que en Colombia se optaba por una atención asistencialista en términos de albergar, alimentar y cuidar a los niños, sobre todo en instituciones del ICBF y en algunas Fundaciones. Los niños que tenían la posibilidad de asistir a instituciones privadas, además del cuidado, lograban tener experiencias orientadas por acciones educativas tendentes a su desarrollo integral.

Sin embargo, y a partir delos análisis adelantados en este trabajo se puede afirmar que hoy en día se continúa, en algunas instituciones, con el cuidado de los niños como principal tarea. El trabajo pedagógico dirigido al desarrollo integral de los niños es escaso, poco programado y algunas veces, desfasado de los procesos de desarrollo. Es importante llamar la atención sobre la necesidad de volver al trabajo pedagógico mediado por el juego como instrumento que permite desarrollos afectivos, psicomotores y cognitivos. Los colegios e instituciones analizadas no tienen en su programación el juego espontaneo, de grupo, ni un espacio curricular para el juego organizado y dirigido.

Asimismo, las rutinas propias de la vida escolar han alejado de las prácticas pedagógicas iniciativas relacionadas con la educación estética, la cual se ha sacrificado por extensos trabajos representados en fichas diarias de trabajo escolar. Muchas de estas fichas son copias de dibujos descontextualizados de la realidad del niño y de su entorno. El trabajo del niño se limita a colorear, recortar y pegar. Este ejercicio desarrollado de manera repetitiva, puede disminuir la motivación y el gusto por el arte, por descubrir y analizar los valores estéticos de la propia cultura, incluido el disfrute por lo literario, por el análisis de su realidad sensible que incluye lo musical, las narrativas, la plástica, lo gráfico, entre otras.
Es conveniente reflexionar sobre la importancia de un proyecto educativo que oriente las intenciones formativas de los educandos y las acciones pertinentes para lograrlo. Es común encontrar en la institución educativa, una programación que consiste en listados de temas para desarrollar en cada mes de la actividad escolar, sin articulación entre sí ni vinculación específica con áreas de desarrollo. En algunas Instituciones la programación hace énfasis en dos grandes campos, lenguaje y matemáticas, desarrollados mediante el trabajo instrumental dejando de lado procesos inherentes al desarrollo del niño. El propósito más importante es que el niño lea, escriba y memorice una buena cantidad de símbolos numéricos.

El ideal de la construcción de un proyecto educativo es que la institución cuente con las directrices pedagógicas que definan sus fines, concepciones, modos de actuación, modelo pedagógico, organización y gestión, entre otros, que le permita desarrollar su labor con un horizonte de sentido sólido que no se vulnere por las presiones externas de la competencia ni por objeciones sin fundamento de padres de familia y la comunidad en general.

\section{Formación docente}

En el análisis se encontró que los docentes encargados de la formación de los niños de las instituciones revisadas, se pueden agrupar en tres grandes categorías con relación a su formación: a) Licenciados en educación, b). profesionales no licenciados y c) Madres comunitarias. Los tres grupos requieren actualización y perfeccionamiento en sus labores educativas. Los dos últimos grupos, requieren además, formación pedagógica y disciplinar que fortalezca las competencias y habilidades requeridas para orientar procesos de desarrollo de la población infantil, tarea compleja y con efectos en el aprendizaje y formación de los niños en su vida escolar futura.

El proceso formativo de las personas responsables de la educación de los niños, debe responderaunaformaciónconbase eneldesarrollo del pensamiento científico, la socioafectividad, 
la comunicación y la creatividad; a entender el juego como una de las estrategias pedagógicas, el disfrute del arte como base del desarrollo de la sensibilidad, y en esencia saber cómo crear ambientes de aprendizaje que permitan a sus educandos, interpretar la realidad y ejercer la autonomía mediante el trabajo en equipos. Un maestro de niños con formación científica, tecnológica, artística, comunicativa, pedagógica y didáctica mediada por la lúdica.

El grupo que merece un trabajo de mayor rigurosidad es el de las madres comunitarias. Constituyen un gran colectivo, con un amplio espectro de acción, lo cual se ve reflejado en un buen número de instituciones que contratan su trabajo, así como las comunidades de niños que están bajo su formación. Dadas sus limitaciones en los dominios de la pedagogía, los conocimientos específicos y las intervenciones didácticas, solo cumplen el papel de cuidadoras, en una población de niños cuyas inteligencias se desarrollan desde la genética en los primeros cinco años.

Existen esfuerzos institucionales para cualificar este colectivo de madres comunitarias y profesionales no licenciados en primera infancia, impartida por instituciones técnicas y de educación superior al cual asiste un porcentaje de madres comunitarias de algunas instituciones. Sin embargo, aún es incipiente.

Con relación a los profesionales no licenciados que trabajan con niños de la primera infancia, necesitan formarse en el ámbito de la pedagogía, las didácticas y la lúdica para que su actuación en el aula tenga la intención pedagógica de las buenas prácticas formativas. Los profesionales no licenciados junto con los licenciados conforman un colectivo que demanda formación permanente vinculada a la práctica profesional, a la investigación, a la comunicación y a la reflexión.

Estos cuatro elementos articulados podrán promover el perfeccionamiento del trabajo docente, si se parte del conocimiento de las necesidades y potencialidades de los niños de la primera infancia, de la formación en investigación del docente, de los manejos fluidos de la comunicación, del disfrute del juego, el arte y la creatividad, que le permita al maestro contrastar las teorías implícitas sobre la educación, la realidad de las familias de los niños, la enseñanza de conocimiento, valores, actitudes y acciones en la construcción de los ambientes de aprendizaje significativo de los niños.

Se requiere una formación de alta calidad para fortalecer el compromiso de quienes fungen como maestros de los niños para que con conocimientos, actitudes y destrezas se comprometan con la búsqueda de variadas alternativas para educar a los niños con las mejores prácticas educativas que fortalezcan su autonomía.

El paso de algunos niños por las instituciones educativas es efímero, para algunos de ellos se constituye en la única oportunidad de estudiar, ir a la institución educativa, de conocer a un maestro, de relacionar sus conocimientos, de trabajar en equipo, de disfrutar materiales de aprendizaje, de comenzar articular conceptos, realidad y solución de problemas con el acompañamiento de un maestra (o), innovador (a), formado para trabajar con niños de la primera infancia.

Existe un problema grave detectado en el estudio, el alto número de niños que no pueden asistir a las instituciones educativas. Según datos del Ministerio de Educación Nacional en el año 2015 de 166.264 niños en edades comprendidas de cero a cuatro años sólo el $6.8 \%$ son atendidos por el sistema educativo. Niños que no van a ninguna institución educativa, por diversos motivos dentro de los cuales se destaca, la no presencia del Estado sobre todo en el área rural; la situación económica de los padres, el nivel de formación de las madres, la imagen de la educación como no portadora de desarrollo inmediato.

La situación del área urbana también es muy grave por los niños que conforman las cinturones de pobreza de la ciudad, el desplazamiento forzoso que afecta la familia pero en forma angustiante a las madres y a los niños; y como otro factor de estudio la misma imagen que tiene la sociedad de los docentes frente al compromiso de la educación. 
La cifra de niños que abandonan la escuela en Santander es alto y pone de manifiesto, un problema que en cambio de disminuir aumenta día a día, por la forma como la calidad de vida se deteriora por los ingresos de los padres para el sostenimiento de los hogares, tanto en el área rural como urbana, además de la desesperación que sufren las familias sin ingreso que viven del diario trascurrir en la informalidad.

Finalmente en este apartado, es importante hacer alusión a la situación laboral de los maestros, profesionales no educadores, y sobre todo las madres comunitarias que viven situaciones difíciles en el campo económico, que se hace evidente en los mismos espacios y condiciones en las cuales son atendidos los niños.

El estudio señala en líneas generales el descontento o insatisfacción por las condiciones laborales en las que se encuentran la mayoría de personas que asumen la educación de la primera infancia. Entrevistados, algunos de ellos consideran que ni el salario, ni las jornadas laborales son apropiados. El común de los salarios es el mínimo, cuando el contrato es de tiempo completo, para medio tiempo, la oferta es la mitad del salario mínimo. La pregunta es qué entiende una institución por medio tiempo, cuando la realidad muestra jornadas de más de cuatro horas diarias.

El problema salarial es complejo vinculado al bienestar de los docentes y al reconocimiento de la profesión. Se requiere un trabajo serio y continúo para que el el Estado, el empleador y la sociedad reconozcan que el empobrecimiento de los docentes también es el empobrecimiento de la educación de los niños.

Con esta realidad se complejiza el tema de la formación, sin embargo puede observarse en los maestros, que a pesar de las circunstancias económicas adversas manifiestan altos niveles de motivación y preocupación por alcanzar mejores niveles de formación científica, pedagógica, ética, comunicativa, y en el manejo de las TIC, donde hay condiciones para aplicarlas, como un reto de la formación de los niños en el siglo XXI.

\section{Vinculación de las familias en el proceso formativo}

Uno de los principales actores en la formación de los niños en forma definitiva es la familia. La presencia de la familia en el proceso formativo es reconocida como un factor ineludible por las bondades y consecuencias que tiene en la formación de los niños, sin embargo se encuentra que ésta responsabilidad no siempre es asumida por los núcleos familiares, en muchos casos es delegada a la institución educativa. El estudio muestra este factor educativo como un faltante en la educación de los menores.

Los padres envían a sus hijos a la institución y no hacen presencia para acompañar a los maestros en la formación de los niños desde el hogar. Entre los factores de mayor relevancia, aportados por las instituciones educativas, se encuentra la situación laboral de los padres, las ocupaciones cuando tienen trabajos en los oficios más humildes y exigentes con horarios que pueden alcanzar hasta doce horas, el desplazamiento al área rural en épocas de cosechas, donde incluso la pareja se desplaza quedando los niños, bajo el cuidado de familiares, cuando no quedan encerrados en sus casas. La situación se torna más dura cuando los padres obtienen los escasos recursos de la economía informal, donde los niños pueden llegar a ser objetos de explotación de variadas formas desde la sexual, la mendicidad o la venta en los semáforos y paraderos de Bucaramanga y su área metropolitana. Cuando los niños tienen la fortuna de la atención de los hogares, el problema se mantiene con un bajo e incluso nulo acompañamiento en las tareas escolares. Es relevante la soledad de los niños en el tiempo que no asisten a la institución educativa pues no hay actividad familiar ni espacios de ocio conjunto.

La condición sociocultural de las familias, es otro factor que afecta en forma muy fuerte el acompañamiento en las responsabilidades escolares y de formación. La mayoría de familias desconocen los procesos orientados por las instituciones, su función como formadora de valores y de hábitos sociales, que en muchos casos entra en contradicción con los mismos 
valores de las familias, Aunque existe voluntad por las instituciones educativas por vincular a los padres de familia en la educación de los niños la realidad muestra fisuras y abismos desde el espacio laboral o la economía informal para el acompañamiento de la familia en la formación de los niños; es casi imposible adelantar talleres de formación de los padres de familia para el acompañamiento en la formación de sus hijos.

En estos escenarios donde se construye la relación: instituciones educativas y familia, se encuentra una profunda depreciación de la educación del niño que se reduce a lo pueda hacer la institución educativa en cabeza de los maestros con formación de licenciados, los profesionales de otros campos del conocimiento, y las madres comunitarias, esta situación genera una serie de riesgos sin el apoyo familiar que inciden en la inserción de los niños en la sociedad.

Trabajar en el campo de las familias es un reto para el avance de los procesos de formación de los niños como seres pertenecientes a una sociedad en constante trasformación, donde los niños son la riqueza de un país, de una región de una misma familia, de una persona llamada a participar en el desarrollo de sí mismo, la familia y la sociedad.

\section{Programas educativos}

El observatorio de la primera infancia, asume los programas educativos como una forma de conocer cómo se están formando los niños y cuáles estrategias didácticas habrá necesidad de crear para formar un niño con grandes potencialidades de aprendizaje en un ambiente privado de las condiciones básicas de alimentación, salud y educación, y con padres que dadas sus necesidades laborales presentan dificultades inmensas para acompañar su formación desde el hogar uno de los grandes retos de los objetivos del milenio.

Los programas educativos ofrecidos a los niños entre cero y seis años en Santander tienen las mismas características de los programas a nivel nacional, primera una desarticulación entre los Ministerios comprometidos en su cumplimiento, por ejemplo Bienestar familiar depende del
Ministerio de Salud en la protección de los niños; la capacitación a las madres comunitarias para la educación de los niños, al parecer es una responsabilidad del Ministerio de Educación, en programas tanto formales como no formales. En este escenario casi todos los Ministerios deberían estar relacionados para un trabajo conjunto y articulado, si bien el problemas esta detectado, y se realizan ingentes esfuerzos de coordinación cada entidad, Bienestar Familiar, SENA, Escuela Normal Superior, ciclos de formación complementaria y Facultades de Educación tributan sus informes, programas y estrategias a sus respetivos ministerios, lo que implica inéditas estrategias para una nueva cosmovisión del Estado acerca de la educación de los niños en Colombia.

Retos y Rutas sobre la educación de la primera infancia

Con el planteamiento hecho sobre las áreas prioritarias, el observatorio considera, que la investigación educativa, relacionada con la educación de los niños, la formación de los docentes, la vinculación de la familia y los programas educativos, es la base para elevar la calidad de la educación en el momento actual.

En la época de la sociedad del conocimiento surgen nuevas relaciones entre el educador y el aprendiz en relación con las cosmovisiones del universo, la revolución en las ciencias, las tecnologías y el arte, hacen de la realidad una complejidad cada vez más explicable desde la interdisdisciplinariedad.

No se puede educar a los niños con los parámetros heredados de la sociedad industrial, la sociedad del conocimiento exige una reinvención de la educación de los niños, una reinvención del educador, una reinvención del aprendiz, una reinvención del conocimiento sobre el hombre, la sociedad, la biodiversidad y universo.

El profesional dedicado a la educación de la primera infancia, debe tener unas características esenciales, desde esta perspectiva asumimos las expuestas por la Asociación Mundial de Educadores Infantiles (AMEI-WAECE) 2016: 
Ética profesional consolidada y una responsabilidad social que le permita formar en sus educandos los más nobles y puros sentimientos hacia lo que les rodea: el medio ambiente, la familia, sus educadores, sus coetáneos, su hogar, su país.

Formación cultural general e integral y un alto nivel creador para iniciar con eficiencia y calidad la formación estética de sus educandos,.

Conocimiento pleno de las particularidades del desarrollo de los niños que forma y educa, tanto desde el punto de vista de su desarrollo fisiológico como psicológico, que le permita una comprensión cabal de sus necesidades, sus intereses y de los requerimientos propios de estos, para lograr un sano desarrollo de la personalidad.

Habilidades pedagógicas necesariase indispensables para dirigir un proceso educativo complejo con niños de las edades entre 0-6 años,

Habilidades para organizar, estructurar y orientar el proceso educativo, en todas sus variantes, dirigido a la participación conjunta de él como educador y de los niños, que constituyen el eje central de su accionar pedagógico.

Habilidades necesarias para realizar un trabajo de atención preventiva y de orientación de la salud y el bienestar de sus niños, que requieren un extremo cuidado para atender todas sus necesidades básicas fundamentales (alimentación, aseo y sueño) y prever los peligros a que están expuestos debido a su vulnerabilidad, fragilidad y poco desarrollo e independencia.

Capacidad para diagnosticar y evaluar el nivel real de competencia de los niños que educa, y la dinámica del proceso de desarrollo de cada uno de ellos de manera sistemática.

Sensibilidad necesaria para comprender la significación de la labor que realiza, y considerarse el máximo responsable de la calidad del aprendizaje y el desarrollo de los niños.

Capacidad para comunicarse con los niños con afecto, bondad e inteligencia, y propiciar en todo tipo de actividad que realice con ellos, las mejores relaciones interpersonales.
Capacidad para investigar y reflexionar acerca del efecto transformador del trabajo educativo que realiza con los niños, la familia y la comunidad.

Capacidad para evaluarse y para someter a la evaluación externa su comportamiento y elevar su nivel cultural, científico y profesional de manera permanente.

Como colofón delas ideas expuestas hasta aquí, nos permitimos presentar a continuación algunos rasgos que complementan los planteamientos de la Asociación Mundial de la Educación Infantil, sobre las condiciones personales del maestro con el propósito de invitar a la reflexión acerca de los retos que supone la educación de los niños y la formación de sus educadores:

1) Interpreta la cultura y el arte como base de su identidad, sensibilidad científica y social. Este rasgo tiene que ver con las teorías, habilidades y actitudes que desarrolla un educador para crear ambientes de aprendizaje que permita desarrollar procesos para compartir los conocimientos y solucionar problemas.

2) Explica la complejidad del universo, la historia del planeta y la historia de las familias de los niños. La sociedad del conocimiento está cruzada por la incertidumbre que coloca en duda las verdades absolutas para los niños de la primera infancia.

3) Revisa, actualiza e innova a partir del PEI de las instituciones de educación preescolar. Es conveniente reflexionar sobre la importancia del PEI, su interpretación, aplicación y planes de mejoramiento en el manejo de las ciencias sociales, ciencias humanas y ciencias naturales. La consilience, La unidad en la diversidad.

4) Entiende que la complejidad, el caos y las crisis son variables que tanto educadores como niños de la primera infancia asumen como rasgos de la educación virtual del siglo XXI.

5) Maneja el pensamiento científico en la solución de problemas. Este rasgo es esencial en la construcción colectiva de la sociedad del conocimiento. Es a través de la articulación teoría 
práctica; mediante la aplicación de los métodos científicos en la solución de problemas que los niños de la primera infancia podrá construir sus bases para aportar valor agregado al mundo científico, tecnológico, o artístico.

6) Aplica innovaciones didácticas en la creación de ambientes de aprendizaje para los niños sobre procesos complejos de la mente humana de acuerdo a las características de los niños. La innovación es un pilar para el desarrollo del ser humano, la biodiversidad y la sociedad.

7) Domina la comprensión de textos y contextos en la toma de decisiones. Este rasgo es vital en el abordaje de la realidad y la toma de decisiones. Una correcta decisión se basa en un sólido conocimiento del ámbito donde desarrollo su quehacer y sus relaciones, su teorización, su prospectiva, su acción.

8) Actúa con ética en los trabajos científicos, culturales y sociales. Este rasgo es indispensable en el actuar de un educador, es el sello de honestidad y ética que requiere las nuevas organizaciones del conocimiento mucho más autónomas flexibles y planetaria.

9) Conoce los ámbitos regional, nacional e internacionaldela primera infancia y las relaciones con otras ciencias. Este rasgo de la identidad le permite el ejercicio de la disciplinariedad, con el fin de producir soluciones creativas.

\section{Referencias}

http://bit.ly/1TaYgCk Asociación Mundial de Educadores Infantiles (AMEI-WAECE)

http://bit.ly/1TLd0lx CEDEFOP. (2008). Habilidades y competencias del siglo XXI para los aprendices del nuevo milenio en los países de la OCDE.

Enseñar creatividad. El espacio educativo.

http://bit.ly/1VW0tC0. Enseñar creatividad. El espacio educativo, Olivia López Martínez Universidad de Murcia - Campus de Espinardo s/n 30100 - Murcia. Correo Electrónico: olivia@um.es

http://www.rae.es/recursos/diccionarios Real Academia de la Lengua Española

http://databases.unesco.org/thessp/ Tesauro de la UNESCO

http://waece.org/diccionario/index.php Diccionario Pedagógico - Asociación Mundial de Educadores Infantiles, waece.org/diccionario/

http://bit.ly/1htogmh Morín Edgar Introducción al Pensamiento Complejo.

http://www.eumed.net/rev/ced/05/fbg2.htm Características generales del niño y la niña de 0 a 6 años, Cuadernos de Educación y Desarrollo, Vol 1, № 5 (julio 2009). Fátima Bejerano.

http://bit.ly/1TT4pQG UNESCO El niño y su desarrollo desde el nacimiento hasta los seis años, París, 1976. 